\title{
$\mathrm{Al}$ 含量对 $\mathrm{Pt}-\mathrm{SO}_{4}^{2-} / \mathrm{ZrO}_{2}-\mathrm{Al}_{2} \mathrm{O}_{3}$ 固体超强酸催化 正戊烷异构化性能的影响
}

\author{
宋 华 ${ }^{1,2}$ * 董鹏飞 ${ }^{1}$ 张 旭 ${ }^{1}$ \\ (1 东北石油大学化学化工学院, 黑龙江 大庆 163318 ; \\ 2 东北石油大学, 石油与天然气化工省重点实验室, 黑龙江 大庆 163318)
}

\begin{abstract}
摘要：通过向 $\mathrm{SO}_{4}^{2-} / \mathrm{ZrO}_{2}$ 催化剂中同时引人适量的 $\mathrm{Pt}$ 和 $\mathrm{Al}_{2} \mathrm{O}_{3}$, 制备出了具有较高催化性能和稳定性的 Pt-SO $\mathrm{ZrO}_{2}-\mathrm{Al}_{2} \mathrm{O}_{3}$ 型固体超强酸催化剂. 以正戊烷异构化反应为探针, 考察了 $\mathrm{Al}$ 含量对催化剂性能的影响; 并采用 $\mathrm{X}$ 射线衍射(XRD)、比表面积测定(BET)、红外(IR)光谱、程序升温还原(TPR)、热重-差热分析(TG-DTA)和氨-程序升 温脱附 $\left(\mathrm{NH}_{3}-\mathrm{TPD}\right)$ 手段对催化剂进行了表征. 结果表明, $\mathrm{Al}$ 能够提高 $\mathrm{ZrO}_{2}$ 的晶化温度, 抑制硫的分解, 增加催化 剂的比表面积, 增强硫氧键的结合, 提高催化剂的还原性能, 增加催化剂的酸强度和酸总量. 当 $\mathrm{Al}_{2} \mathrm{O}_{3}$ 含量(质量 分数, $w$ ) 为 $5.0 \%$ 时, $\mathrm{Pt}-\mathrm{SO}_{4}^{2-} / \mathrm{ZrO}_{2}-\mathrm{Al}_{2} \mathrm{O}_{3}$ 固体超强酸催化剂的催化活性最好, 在 $100 \mathrm{~h}$ 内异戊烷收率可稳定在 $52.0 \%$ 以上, 选择性在 $98.2 \%$ 以上.
\end{abstract}

关键词：固体超强酸; 催化剂; $\mathrm{SO}_{4}^{2-} / \mathrm{ZrO}_{2} ;$ 异构化; $\mathrm{Al}_{2} \mathrm{O}_{3}$ 含量； $\mathrm{Pt}$ 中图分类号：0643

\section{Effect of Al Content on the $\boldsymbol{n}$-Pentane Isomerization of the Solid Superacid Pt-SO ${ }_{4}^{2-} / \mathrm{ZrO}_{2}-\mathrm{Al}_{2} \mathbf{O}_{3}$}

\author{
SONG Hua ${ }^{1,2, *} \quad$ DONG Peng-Fei ${ }^{1} \quad$ ZHANG Xu ${ }^{1}$ \\ ( ${ }^{1}$ College of Chemistry and Chemical Engineering, Northeast Petroleum University, Daqing 163318, Heilongjing Province, \\ P. R. China; ${ }^{2}$ Heilongjiang Provincial Key Laboratory of Chemical Engineering of Oil and Gas, \\ Northeast Petroleum University, Daqing 163318, Heilongjiang Province, P. R. China)
}

\begin{abstract}
Highly active and stable $\mathrm{Pt}-\mathrm{SO}_{4}^{2-} / \mathrm{ZrO}_{2}-\mathrm{Al}_{2} \mathrm{O}_{3}$ solid superacid catalysts were prepared by simultaneously introducing appropriate amounts of $\mathrm{Pt}$ and $\mathrm{Al}_{2} \mathrm{O}_{3}$ into a $\mathrm{SO}_{4}^{2-} / \mathrm{ZrO}_{2}$ catalyst. The effect of $\mathrm{Al}$ content on the performance of the catalysts was studied using $n$-pentane isomerization as a probe reaction and the catalysts were characterized by $\mathrm{X}$-ray diffraction (XRD), specific surface area measurements (BET), infrared (IR) spectroscopy, temperature-programmed reduction (TPR), thermogravimetry-differential thermal analysis (TG-DTA), and $\mathrm{NH}_{3}$ temperature-programmed desorption $\left(\mathrm{NH}_{3}\right.$-TPD). The results show that $\mathrm{Al}$ can increase the crystallization temperature of $\mathrm{ZrO}_{2}$ and inhibit the decomposition of sulfur. Al can also increase the surface area of the catalyst, strengthen the combination between $\mathrm{S}$ and $\mathrm{O}$, improve the redox performance of the catalyst and increase the acid strength and the acidity of the catalyst. The catalytic activity of the Pt-SO $\mathrm{SO}_{4}^{2-} / \mathrm{ZrO}_{2}-\mathrm{Al}_{2} \mathrm{O}_{3}$ solid superacid catalyst with a $\mathrm{Al}_{2} \mathrm{O}_{3}$ mass fraction of $5.0 \%$ was found to be the best. The isopentane yield was stable above $52.0 \%$ and the selectivity was higher than $98.2 \%$ within $100 \mathrm{~h}$.
\end{abstract}

Key Words : Solid superacid; Catalyst; $\quad \mathrm{SO}_{4}^{2-} / \mathrm{ZrO}_{2} ;$ Isomerization; $\mathrm{Al}_{2} \mathrm{O}_{3}$ content; $\mathrm{Pt}$

Received: December 23, 2009; Revised: March 9, 2010; Published on Web: May 13, 2010.

"Corresponding author. Email: songhua@dqpi.edu.cn; Tel: +86-459-6503167.

The project was supported by the Science and Technological Innovation Foundation of PetroChina Company Limited (07-06D-01-04-03-02). 中国石油天然气股份有限公司科技风险创新基金(07-06D-01-04-03-02)资助项目

(C) Editorial office of Acta Physico-Chimica Sinica 
固体超强酸具有不污染环境、选择性好、无腐蚀 性、制备方法简便等优点, 在酸催化作用中, 固体超 强酸克服了液体酸催化剂的许多弊端, 对异构化、烷 基化、脱水及酯化等反应具有很高的催化活性, 受到 人们的普遍重视 ${ }^{[1-2]}$. 固体超强酸中的 $\mathrm{SO}_{4}^{2-} / \mathrm{M}_{x} \mathrm{O}_{y}(\mathrm{M}=$ $\mathrm{Fe} 、 \mathrm{Hf} 、 \mathrm{Ti} 、 \mathrm{Sn} 、 \mathrm{Zr}$ 等)型固体超强酸因具有不含卤离 子、酸强度高、反应温度低、催化活性高等突出优点, 并对绝大部分酸催化反应都表现出良好的反应活性 和选择性, 成为目前催化领域研究的热点之一 ${ }^{[3]}$. 高 滋等 ${ }^{[4]}$ 将 $\mathrm{SO}_{4}^{2-} / \mathrm{ZrO}_{2}$ 应用于正构烷烃的异构化反应, 但 $\mathrm{SO}_{4}^{2-} / \mathrm{M}_{x} \mathrm{O}_{y}$ 类超强酸存在稳定性差、寿命较短等缺 点, 因此, 需要对 $\mathrm{SO}_{4}^{2-} / \mathrm{M}_{x} \mathrm{O}_{y}$ 型固体超强酸催化剂进 行改性, 以提高其催化性能和使用寿命.

本文用 $\mathrm{Al}(\mathrm{OH})_{3}$ 与 $\mathrm{Zr}(\mathrm{OH})_{4}$ 共沉淀法制备铝促 进的硫酸根稳定的氧化锆催化剂 $\mathrm{SO}_{4}^{2-} / \mathrm{ZrO}_{2}-\mathrm{Al}_{2} \mathrm{O}_{3}$, 并通过向 $\mathrm{SO}_{4}^{2-} / \mathrm{ZrO}_{2}-\mathrm{Al}_{2} \mathrm{O}_{3}$ 催化剂中同时引人适量 $\mathrm{Pt}$ 制备出具有较高催化性能和高稳定性的 $\mathrm{Pt}-\mathrm{SO}_{4}^{2-}$ $\mathrm{ZrO}_{2}-\mathrm{Al}_{2} \mathrm{O}_{3}$ 型固体超强酸催化剂, 同时通过表征等 手段研究制备条件对其正戊烷异构化性能的影响.

\section{1 实验部分}

\section{1 原料及试剂}

$\mathrm{ZrOCl}_{2} \cdot 8 \mathrm{H}_{2} \mathrm{O}$ (上海润捷化学试剂有限公司), $\mathrm{Al}\left(\mathrm{NO}_{3}\right)_{3} \cdot 9 \mathrm{H}_{2} \mathrm{O}$ (天津市纵横化工试剂分公司), $\mathrm{H}_{2} \mathrm{PtCl}_{6} \cdot$ $6 \mathrm{H}_{2} \mathrm{O}$ (沈阳市金科试剂厂), $\mathrm{H}_{2} \mathrm{SO}_{4}$ (哈尔滨市化工试 剂厂), 正戊烷, $\mathrm{HNO}_{3}$, 均为分析纯; 使用去离子水 (蒸馏水), 浓氨水(质量浓度为 $25 \%$ ).

\section{2 催化剂的制备}

催化剂制备用共沉淀法: (1) 将 $\mathrm{ZrOCl}_{2} \cdot 8 \mathrm{H}_{2} \mathrm{O}$ 和 一定量的 $\mathrm{Al}\left(\mathrm{NO}_{3}\right)_{3} \cdot 9 \mathrm{H}_{2} \mathrm{O}$ 溶于蒸馏水, 用氨水滴定 至 $\mathrm{pH}=9.3$. 沉淀陈化 $24 \mathrm{~h}$, 反复洗涤至无 $\mathrm{Cl}^{-}, 110$ ${ }^{\circ} \mathrm{C}$ 干燥, 研磨至 120 目以上, 得到催化剂前驱体; (2) 将该前驱体粉末用 $0.5 \mathrm{~mol} \cdot \mathrm{L}^{-1} \mathrm{H}_{2} \mathrm{SO}_{4}$ 溶液浸渍 $1 \mathrm{~h}$, 烘干 $4 \mathrm{~h}$ 后, 用计算量的 $\mathrm{H}_{2} \mathrm{PtCl}_{6}$ 溶液等体积浸渍 4 $\mathrm{h}$, 使 $\mathrm{Pt}$ 的负载量为 $0.10 \%$, 然后烘干 $4 \mathrm{~h}$, 加人田菁 粉 (田菁粉与催化剂的质量比为 0.05 ), 挤条, $650{ }^{\circ} \mathrm{C}$ 焙烧, 制得 $\mathrm{Al}_{2} \mathrm{O}_{3}$ 含量 $(w)$ 分别为 $0 \% 、 2.5 \% 、 5.0 \%$ 、 $10.0 \% 、 30.0 \%$ 的催化剂, 其 $\mathrm{Pt}$ 质量分数均为 $0.10 \%$.

\section{3 催化剂的活性评价}

催化剂的活性评价采用高压微反-色谱联合装 置, 原料为正戊烷. 将 $5 \mathrm{~g}$ 催化剂装人反应器中, 用 $\mathrm{H}_{2}$ 在 $300{ }^{\circ} \mathrm{C}$ 还原 $3 \mathrm{~h}$ 后, 在反应压力 $2.0 \mathrm{MPa}$ 、氢烃 摩尔比 4:1、质量空速(WHSV) $1.0 \mathrm{~h}^{-1}$ 不变的工艺条
件下进行异构化反应, 产物用气相色谱在线分析.

\section{4 实验仪器}

$\mathrm{X}$ 射线衍射(XRD)表征在日本理学公司 $\mathrm{D} / \mathrm{max}-$ $2200 X$ 射线衍射仪上进行, $\mathrm{Cu}$ 靶, $K_{\alpha}$ 线, 扫描范围 $10^{\circ}-80^{\circ}$. 傅里叶变换红外(FT-IR)光谱分析采用德国 布鲁克光谱仪器公司的 Tensor 27 傅里叶红外光谱 仪, 扫描范围 400-4000 $\mathrm{cm}^{-1}, \mathrm{KBr}$ 压片. 比表面积 (BET)测试采用美国 Micromeritics ASAP2400 测定 仪, 样品在 $250{ }^{\circ} \mathrm{C}, 1.3 \mathrm{~Pa}$ 下预处理 $4 \mathrm{~h}$. 热重-差热分 析(TG-DTA)采用美国 Perkin Elmer 公司生产的 DuPont 2100 型热分析仪, 空气环境, 升温速率为 10 ${ }^{\circ} \mathrm{C} \cdot \mathrm{min}^{-1}$, 样品质量为 $0.2000 \mathrm{~g}$. 程序升温还原 $(\mathrm{TPR})$ 由美国康塔公司生产的 ChemBET3000 化学吸附仪 测定, 采用热导池做检测器, 气体流速为 $20 \mathrm{~mL}$. $\mathrm{min}^{-1}$, 升温速率为 $5{ }^{\circ} \mathrm{C} \cdot \mathrm{min}^{-1}$. 氨程序升温脱附 $\left(\mathrm{NH}_{3-}\right.$ TPD)由美国康塔公司生产的 ChemBET3000 化学吸 附仪测定, 采用热导池做检测器, He 做载气, 气体流 速为 $20 \mathrm{~mL} \cdot \mathrm{min}^{-1}$, 升温速率为 $5{ }^{\circ} \mathrm{C} \cdot \mathrm{min}^{-1}$.

\section{2 结果与讨论}

\section{$2.1 \mathrm{Al}_{2} \mathrm{O}_{3}$ 质量分数对催化剂性能的影响}

\subsection{1 不同 $\mathrm{Al}_{2} \mathrm{O}_{3}$ 含量催化剂的 XRD 分析}

图 1、图 2 分别为 $650 、 700{ }^{\circ} \mathrm{C}$ 焙烧得到的不同 $\mathrm{Al}_{2} \mathrm{O}_{3}$ 质量分数的催化剂的 XRD 谱图. 从图 1 、图 2 可看出, 谱图中没有出现 $\mathrm{Zr}\left(\mathrm{SO}_{4}\right)_{2}$ 晶相峰 $\left(2 \theta \approx 1.9^{\circ}\right.$, $\left.2.1^{\circ}, 2.9^{\circ}\right)$, 未添加铝的催化剂既含有四方晶相 $(2 \theta \approx$ $\left.30.5^{\circ}, 35.5^{\circ}, 50.8^{\circ}, 60.4^{\circ}\right)$ 又含有单斜晶相 $\left(2 \theta \approx 24.2^{\circ}\right.$, $28.2^{\circ}, 31.5^{\circ}, 34.2^{\circ}, 41.0^{\circ}, 45.0,55.8^{\circ}$ ), 添加铝以后 的催化剂无明显的单斜晶相, 说明铝的加人可以稳

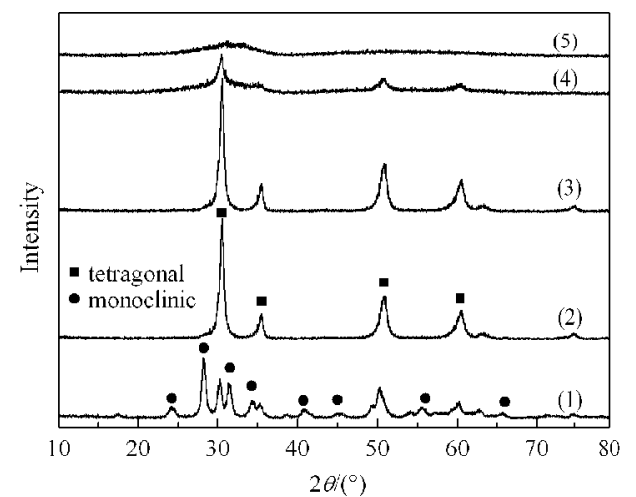

图 $1650{ }^{\circ} \mathrm{C}$ 焙烧 $\mathrm{Al}_{2} \mathrm{O}_{3}$ 质量分数不同的 $\mathrm{Pt}^{-\mathrm{SO}_{4}^{2-} / \mathrm{ZrO}_{2}-}$ $\mathrm{Al}_{2} \mathrm{O}_{3}$ 催化剂 $\mathrm{XRD}$ 图

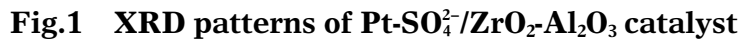
with different $\mathrm{Al}_{2} \mathrm{O}_{3}$ mass fractions at $650{ }^{\circ} \mathbf{C}$ $w\left(\mathrm{Al}_{2} \mathrm{O}_{3}\right)$ : (1) $0 \%$; (2) $2.5 \%$; (3) $5.0 \%$; (4) $10.0 \%$; (5) $30.0 \%$ 


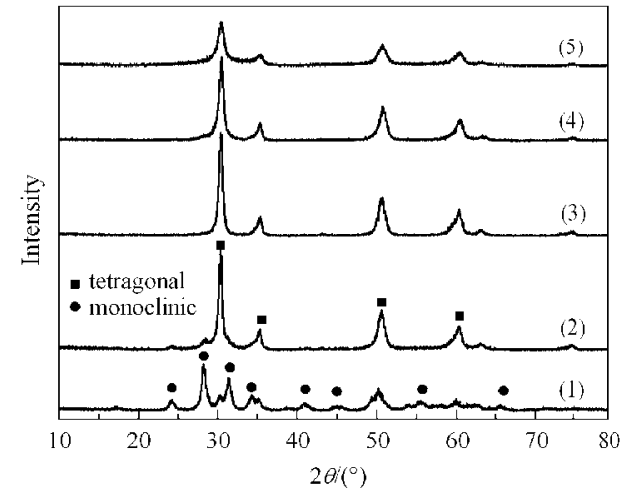

图 $2700{ }^{\circ} \mathrm{C}$ 焙烧 $\mathrm{Al}_{2} \mathrm{O}_{3}$ 质量分数不同的 $\mathrm{Pt}^{-} \mathrm{SO}_{4}^{2-I}$ $\mathrm{ZrO}_{2}-\mathrm{Al}_{2} \mathrm{O}_{3}$ 催化剂 XRD 图

Fig. 2 XRD patterns of $\mathrm{Pt}_{-} \mathrm{SO}_{4}^{2-} / \mathrm{ZrO}_{2}-\mathrm{Al}_{2} \mathrm{O}_{3}$ catalyst with different $\mathrm{Al}_{2} \mathbf{O}_{3}$ mass fractions at $700{ }^{\circ} \mathrm{C}$ $w\left(\mathrm{Al}_{2} \mathrm{O}_{3}\right)$ : (1) $0 \%$; (2) $2.5 \%$; (3) $5.0 \%$;(4) $10.0 \%$; (5) $30.0 \%$

定氧化锆的四方晶相结构, 延缓 $\mathrm{ZrO}_{2}$ 由 $\mathrm{T}$ 相 (四方 相)向 $\mathrm{M}$ 相(单斜相)的转变. 氧化锆在焙烧过程中的 晶化是通过表面扩散机理进行的, 因此晶粒长大的 难易与氧化锆周围的环境有关. 随着焙烧脱水和晶 化的进行, 非晶态的氧化锆首先在粉粒中的有利部 位析晶, 并随温度升高而成核长大. 与纯氧化锆相 比, 氢氧化锆和氢氧化铝共沉淀后, 由于氢氧化铝的 存在, 氧化锆粉粒相均匀接触的数量显著减少, 氧化 锆直接接触并长大必然受到很大的限制, 氧化锆粉 粒需越过周围的氢氧化铝扩散至邻近的氧化锆粉 粒, 迁移距离增加, 扩散受到阻碍. 在焙烧中氢氧化 锆的烃基与氢氧化铝的烃基可能发生缩合并逐步形 成 $\mathrm{Zr}-\mathrm{O}-\mathrm{Al}$ 键, 这样氢氧化铝经过高温焙烧后得 到的氧化铝对氧化锆会起到一个 “针定”作用, 限制 了氧化锆的迁移扩散 ${ }^{[5]}$. 这些因素都会使氧化锆的 晶化变得困难, 导致晶化温度提高. 焙烧温度为 650 ${ }^{\circ} \mathrm{C}$ 时, $\mathrm{Al}_{2} \mathrm{O}_{3}$ 质量分数为 $2.5 \%$ 和 $5.0 \%$ 的催化剂主要 为四方晶相, 而 $\mathrm{Al}_{2} \mathrm{O}_{3}$ 质量分数为 $10.0 \% 、 30.0 \%$ 的 催化剂在 $2 \theta \approx 30^{\circ}$ 处出现较宽的弥散峰, 其晶相基 本为无定形结构, 抑制了四方晶相的形成; 焙烧温 度提高到 $700{ }^{\circ} \mathrm{C}$ 时, $\mathrm{Al}_{2} \mathrm{O}_{3}$ 质量分数为 $10.0 \% 、 30.0 \%$ 的催化剂的无定形结构逐渐向四方晶相转变.

\subsection{2 不同 $\mathrm{Al}_{2} \mathrm{O}_{3}$ 含量催化剂的 IR 分析}

图 3 为焙烧温度为 $650{ }^{\circ} \mathrm{C}$ 时, $\mathrm{Al}_{2} \mathrm{O}_{3}$ 质量分数分 别为 $0 \%$ 和 $5.0 \%$ 的 $\mathrm{Pt}-\mathrm{SO}_{4}^{2-} / \mathrm{ZrO}_{2}-\mathrm{Al}_{2} \mathrm{O}_{3}$ 催化剂的红 外光谱图. 一般认为, 氧化物表面结合的 $\mathrm{SO}_{4}^{2-}$ 有 3 种存在状态: 单配位、鳌合双配位和桥式双配位, 鳌 合双配位或桥式双配位可由表面 $\mathrm{SO}_{4}^{2-}$ 最高反对称 振动的特征吸收峰的位置决定, 当该吸收峰在 1200

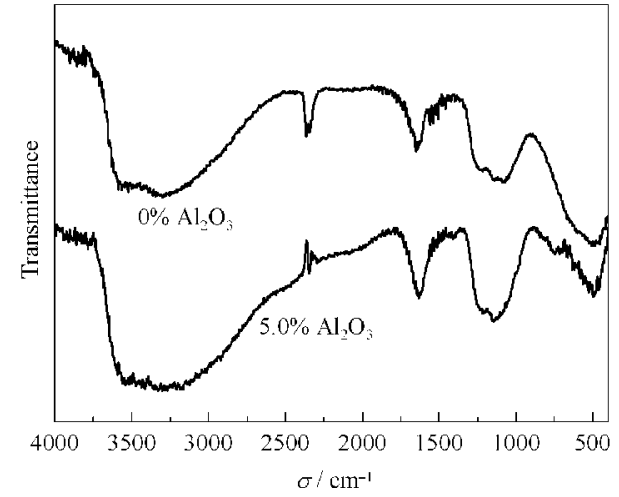

图 3 不同 $\mathrm{Al}_{2} \mathrm{O}_{3}$ 质量分数的 $\mathrm{Pt}-\mathrm{SO}_{4}^{2-} / \mathrm{ZrO}_{2}-\mathrm{Al}_{2} \mathrm{O}_{3}$ 催化剂的 IR 光谱图

Fig.3 IR spectra of $\mathrm{Pt}-\mathrm{SO}_{4}^{2-} / \mathrm{ZrO}_{2}-\mathrm{Al}_{2} \mathrm{O}_{3}$ catalysts with different $\mathrm{Al}_{2} \mathrm{O}_{3}$ mass fractions

$\mathrm{cm}^{-1}$ 以上时为鳌合双配位吸附, 在 $1200 \mathrm{~cm}^{-1}$ 以下时 为桥式双配位吸附.

由图 3 可以看出, 催化剂在 $1639 \mathrm{~cm}^{-1}$ 处的吸收 峰是表面复合氧化物吸附水的 $\mathrm{O}-\mathrm{H}$ 变形振动峰; 催化剂在 $1070 \mathrm{~cm}^{-1}$ 处 $\left(\mathrm{Al}_{2} \mathrm{O}_{3}\right.$ 质量分数为 $\left.0 \%\right)$ 和 1150 $\mathrm{cm}^{-1}$ 处 $\left(\mathrm{Al}_{2} \mathrm{O}_{3}\right.$ 质量分数为 $\left.5.0 \%\right)$ 的吸收峰为 $\mathrm{O}-\mathrm{S}-\mathrm{O}$ 键的对称伸缩振动吸收峰 ${ }^{[6]} ; 1133-1400 \mathrm{~cm}^{-1}$ 处的 吸收峰为 $\mathrm{S}-\mathrm{O}$ 的反对称伸缩振动吸收峰 ${ }^{[6]}$, 是 $\mathrm{SO}_{4}^{2-}$ 促进型超强酸的特征吸收峰, 表明催化剂表面的 $\mathrm{SO}_{4}^{2-}$ 以鳌合双配位方式吸附在金属离子上. 由图 3 还可以看出, $\mathrm{Al}_{2} \mathrm{O}_{3}$ 质量分数为 $5.0 \%$ 的催化剂䢃裂 强度明显高于不含 $\mathrm{Al}_{2} \mathrm{O}_{3}$ 的催化剂, 而且前者的特 征峰面积明显大于后者, 表明 $\mathrm{Al}_{2} \mathrm{O}_{3}$ 的加人使催化 剂具有更高的酸强度和更多的表面活性中心; 又由 于 $\mathrm{SO}_{4}^{2-}$ 中 $\mathrm{O}$ 的吸电子倾向, $\mathrm{Al}_{2} \mathrm{O}_{3}$ 的加人使 $\mathrm{ZrO}_{2}$ 中 原本带有 $\delta$ 正电荷的 $\mathrm{Zr}(\mathrm{IV})$ 带有更多的正电荷, 从 $\mathrm{O}-\mathrm{S}-\mathrm{O}$ 键吸收峰的位置看, 加人 $\mathrm{Al}_{2} \mathrm{O}_{3}$ 后键级升 高, 可以判断 $\mathrm{Al}_{2} \mathrm{O}_{3}$ 的加人使催化剂表现出更强的 Lewis 酸性 ${ }^{[7]}$.

\subsection{3 不同 $\mathrm{Al}_{2} \mathrm{O}_{3}$ 含量催化剂的 $\mathrm{BET}$ 表征}

不同 $\mathrm{Al}_{2} \mathrm{O}_{3}$ 质量分数的催化剂的比表面积和孔 结构表征结果见表 1 . 由表 1 可知, 随着催化剂中 $\mathrm{Al}_{2} \mathrm{O}_{3}$ 质量分数的增加, 催化剂的比表面积逐渐增 大, $\mathrm{Al}_{2} \mathrm{O}_{3}$ 质量分数由 $2.5 \%$ 增加到 $30.0 \%$ 时, 催化剂 的比表面积由 $94 \mathrm{~m}^{2} \cdot \mathrm{g}^{-1}$ 增加到 $139 \mathrm{~m}^{2} \cdot \mathrm{g}^{-1}$. 随着催 化剂中 $\mathrm{Al}_{2} \mathrm{O}_{3}$ 质量分数的增加, 催化剂孔径有所减 小, 但变化不大; 孔体积有明显增大的趋势, $\mathrm{Al}_{2} \mathrm{O}_{3}$ 质 量分数为 $30.0 \%$ 的催化剂的孔体积 $\left(0.210 \mathrm{~cm}^{3} \cdot \mathrm{g}^{-1}\right)$ 比 $\mathrm{Al}_{2} \mathrm{O}_{3}$ 质量分数为 $2.5 \%$ 的孔体积 $\left(0.103 \mathrm{~cm}^{3} \cdot \mathrm{g}^{-1}\right)$ 增加 一倍多. 引人 $\mathrm{Al}_{2} \mathrm{O}_{3}$ 可以降低催化剂的生产成本, 且 
表 1 不同 $\mathrm{Al}_{2} \mathrm{O}_{3}$ 质量分数的 $\mathrm{Pt}-\mathrm{SO}_{4}^{2-} / \mathrm{ZrO}_{2}-\mathrm{Al}_{2} \mathrm{O}_{3}$ 催化剂 的比表面积和孔结构

Table 1 Specific surface area and pore structure of $\mathrm{Pt}-\mathrm{SO}_{4}^{2-} / \mathrm{ZrO}_{2}-\mathrm{Al}_{2} \mathrm{O}_{3}$ catalysts with different $\mathrm{Al}_{2} \mathrm{O}_{3}$ mass fractions

\begin{tabular}{cccc}
\hline$w\left(\mathrm{Al}_{2} \mathrm{O}_{3}\right)(\%)$ & $S /\left(\mathrm{m}^{2} \cdot \mathrm{g}^{-1}\right)$ & $d / \mathrm{nm}$ & $V /\left(\mathrm{cm}^{3} \cdot \mathrm{g}^{-1}\right)$ \\
\hline 0.0 & 80 & 4.96 & 0.094 \\
2.5 & 94 & 4.93 & 0.103 \\
5.0 & 108 & 4.88 & 0.122 \\
10.0 & 127 & 4.70 & 0.150 \\
30.0 & 139 & 4.57 & 0.210 \\
\hline
\end{tabular}

适量的 $\mathrm{Al}_{2} \mathrm{O}_{3}$ 可以分散和稳定氧化物表面的 $\mathrm{SO}_{4}^{2-}$, 并能增大催化剂的比表面积, 从而增加有效酸性位 的数目. 但是, $\mathrm{ZrO}_{2}$ 能够与 $\mathrm{SO}_{4}^{2-}$ 作用形成超强酸位, 所以, $\mathrm{Al}_{2} \mathrm{O}_{3}$ 加人量过大, 催化剂中 $\mathrm{ZrO}_{2}$ 的质量分数 相对较低, 活性中心数目减少.

\subsection{4 不同 $\mathrm{Al}_{2} \mathrm{O}_{3}$ 含量催化剂的 TG-DTA 分析}

图 4 给出了 $\mathrm{Al}_{2} \mathrm{O}_{3}$ 质量分数为 $5.0 \%$ 的不经焙 烧的催化剂的差热分析(DTA)和热重(TG)分析曲线. DTA 曲线上 $300{ }^{\circ} \mathrm{C}$ 前的吸热峰, 对应 TG 曲线上物 理吸附水和水合氧化物结构水的脱除; DTA 曲线上 $600{ }^{\circ} \mathrm{C}$ 以后的放热峰是由于氧化锆发生晶化转化过 程而引起的. $600{ }^{\circ} \mathrm{C}$ 以后的吸热峰归属于 $\mathrm{SO}_{4}^{2-}$ 受热 分解引起. 热重 $\mathrm{TG}$ 曲线分为 3 段: 第 1 段是快速失 重区, 从室温至 $200{ }^{\circ} \mathrm{C}, \mathrm{TG}$ 曲线随温度升高快速下 降, 主要失去的是催化剂表面较多的物理吸附水; 第 2 段是 200-650 ${ }^{\circ} \mathrm{C}$ 相对恒重区, TG 曲线变化不 大, 主要失去的是催化剂表面较少的吸附水, 少量游 离的酸等; 第 3 段是 $650-800{ }^{\circ} \mathrm{C}$, 失重的主要是与 $\mathrm{ZrO}_{2}$ 形成超强酸结构的硫. 试验过程中在 $300{ }^{\circ} \mathrm{C}$ 前 将尾气通人无水硫酸铜和品红试液, 发现无水硫酸 铜变蓝, 而品红试液不褪色; 而在高温 $600{ }^{\circ} \mathrm{C}$ 下将尾

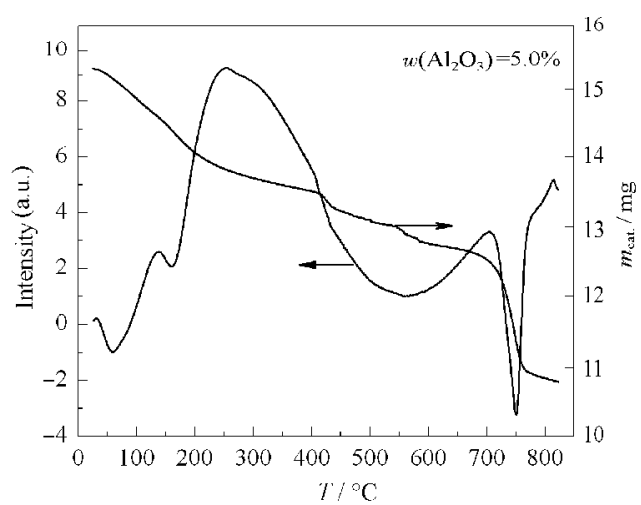

图 4 不经焙烧的 $\mathrm{Pt}-\mathrm{SO}_{4}^{2-} / \mathrm{ZrO}_{2}-\mathrm{Al}_{2} \mathrm{O}_{3}$ 催化剂的差热分析 (DTA)和热重(TG)分析

Fig.4 TG-DTA curves of non-calcined $\mathrm{Pt}^{-\mathrm{SO}_{4}^{2-}} / \mathrm{ZrO}_{2}$ $\mathbf{A l}_{2} \mathbf{O}_{3}$ catalyst
气通人无水硫酸铜, 基本不变色, 而品红试液红色退 去, 加热后红色复现(这点可以排除氯离子的干扰), 说明有 $\mathrm{SO}_{2}$ 气体的产生, 而这一现象与 Vijay 等 ${ }^{[8]}$ 通 过高分辨电子轰击质谱(EI-MS)等手段检测到的硫 酸的实验相吻合. 固体超强酸 TG 谱图说明了 680 ${ }^{\circ} \mathrm{C}$ 是硫与 $\mathrm{ZrO}_{2}$ 成键被破坏的临界温度, 因此, 催化 剂焙烧温度不宜超过 $680{ }^{\circ} \mathrm{C}$.

图 5 为 $\mathrm{Al}_{2} \mathrm{O}_{3}$ 质量分数为 $0 \%$ 和 $5.0 \%$ 的不经焙 烧的催化剂热重图. 由图 5 可以看出, 不含 $\mathrm{Al}_{2} \mathrm{O}_{3}$ 的 催化剂在 $300{ }^{\circ} \mathrm{C}$ 左右时有一定的失重, 在 $500{ }^{\circ} \mathrm{C}$ 左 右时失重非常明显; 而 $\mathrm{Al}_{2} \mathrm{O}_{3}$ 质量分数为 $5.0 \%$ 的催 化剂当温度升至 $650{ }^{\circ} \mathrm{C}$ 时, 有一定的失重, 并且在 $760{ }^{\circ} \mathrm{C}$ 之后失重缓慢. 由此可知, $\mathrm{Al}_{2} \mathrm{O}_{3}$ 的加人能够 提高催化剂中硫酸根的分解温度, 抑制硫酸根的分 解, 增加了负载在催化剂上硫的热稳定性, 而造成这 种现象的原因可能是因为 $\mathrm{Al}_{2} \mathrm{O}_{3}$ 掺杂进人 $\mathrm{ZrO}_{2}$ 后, 由于 $\mathrm{Al}_{2} \mathrm{O}_{3}$ 和 $\mathrm{ZrO}_{2}$ 的粒子半径不同, 半径大小不同 的粒子相互取代造成晶格参数发生很大变化, 其中 相当多的 $\mathrm{SO}_{4}^{2-}$ 占据了 $\mathrm{Al}_{2} \mathrm{O}_{3}$ 表面畸变八面体位置, 而 $\mathrm{SO}_{4}^{2-}$ 在这个位置上较难分解 ${ }^{[9]}$, 所以 $\mathrm{Al}_{2} \mathrm{O}_{3}$ 的加 人能够提高催化剂中硫酸根的分解温度.

\subsection{5 不同 $\mathrm{Al}_{2} \mathrm{O}_{3}$ 含量催化剂的 TPR 分析}

图 6 为在 $\mathrm{H}_{2}$ 气氛中温度在 $100-800{ }^{\circ} \mathrm{C}$ 下不同 $\mathrm{Al}_{2} \mathrm{O}_{3}$ 质量分数的 Pt- $\mathrm{SO}_{4}^{2-} / \mathrm{ZrO}_{2}-\mathrm{Al}_{2} \mathrm{O}_{3}$ 催化剂的 TPR 图. 由图 6 可知, $\mathrm{Al}_{2} \mathrm{O}_{3}$ 质量分数为 $5.0 \%$ 的催化剂还 原峰温度为 $605{ }^{\circ} \mathrm{C}, \mathrm{Al}_{2} \mathrm{O}_{3}$ 质量分数为 $2.5 \%$ 的催化 剂还原峰温度为 $640{ }^{\circ} \mathrm{C}$, 而含 $\mathrm{Al}_{2} \mathrm{O}_{3}$ 为 $0 \%$ 的催化剂 的还原峰温度为 $670{ }^{\circ} \mathrm{C}$, 比 $\mathrm{Al}_{2} \mathrm{O}_{3}$ 为 $5.0 \%$ 的催化剂 升高了 $65{ }^{\circ} \mathrm{C}$ 左右. 试验中在 $600-700{ }^{\circ} \mathrm{C}$ 时在尾气中 检测到 $\mathrm{H}_{2} \mathrm{~S}$ 和水蒸气, 表明 $\mathrm{H}_{2}$ 在高温下将载体上负

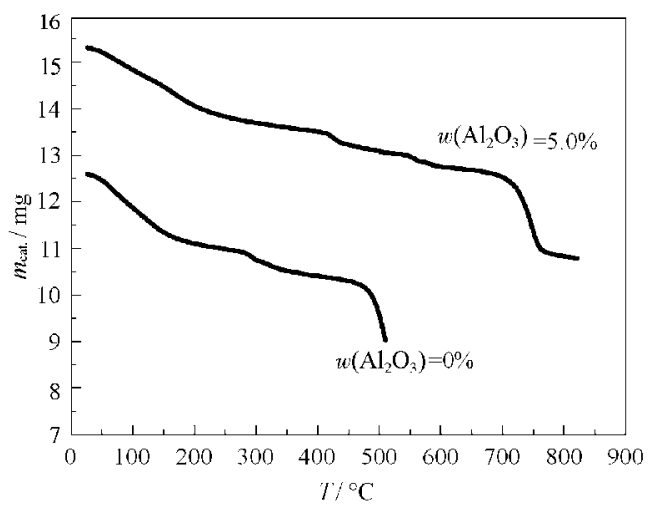

图 5 不同 $\mathrm{Al}_{2} \mathrm{O}_{3}$ 质量分数的不经焙烧的 $\mathrm{Pt}^{-\mathrm{SO}_{4}^{2-} / \mathrm{ZrO}_{2^{-}}}$ $\mathrm{Al}_{2} \mathrm{O}_{3}$ 催化剂 TG 图

Fig.5 TG curves of non-calcined Pt-SO $\mathrm{SO}_{4}^{2-} / \mathrm{ZrO}_{2}-\mathrm{Al}_{2} \mathrm{O}_{3}$ catalyst with different $\mathrm{Al}_{2} \mathrm{O}_{3}$ mass fractions 


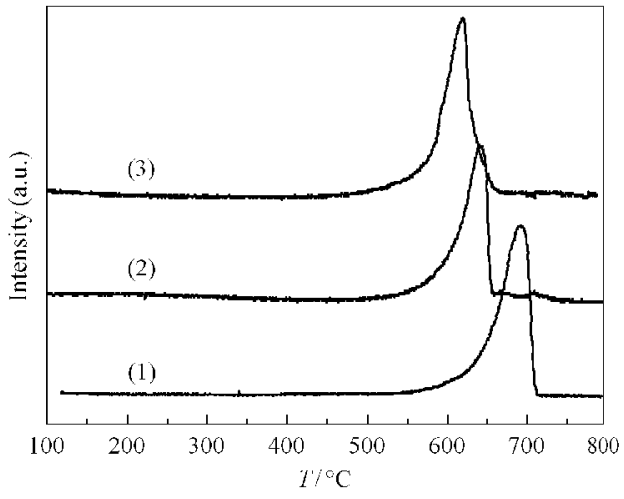

图 6 不同 $\mathrm{Al}_{2} \mathrm{O}_{3}$ 质量分数的 $\mathrm{Pt}-\mathrm{SO}_{4}^{2-} / \mathrm{ZrO}_{2}-\mathrm{Al}_{2} \mathrm{O}_{3}$ 催化剂的 TPR 谱图

Fig.6 TPR profiles of $\mathrm{Pt}-\mathrm{SO}_{4}^{2-} / \mathrm{ZrO}_{2}-\mathrm{Al}_{2} \mathrm{O}_{3}$ catalysts with different $\mathrm{Al}_{2} \mathbf{O}_{3}$ mass fractions $w\left(\mathrm{Al}_{2} \mathrm{O}_{3}\right)$ : (1) $0 \%$; (2) $2.5 \%$; (3) $5.0 \%$

载的 $\mathrm{SO}_{4}^{2-}$ 还原为 $\mathrm{H}_{2} \mathrm{~S}$ 和 $\mathrm{H}_{2} \mathrm{O} . \mathrm{Al}_{2} \mathrm{O}_{3}$ 能够使 $\mathrm{SO}_{4}^{2-}$ 的还 原温度降低, 使耗氢峰左移的原因可能是: (1) $\mathrm{Al}_{2} \mathrm{O}_{3}$ 和 $\mathrm{ZrO}_{2}$ 进行掺杂后晶格形成固溶体, 粒子价态不 同, 配位数就不同, 为了保持晶格内电荷守恒, 氧离 子从晶格中解析出来形成氧空穴, 同时 $\mathrm{Al}_{2} \mathrm{O}_{3}$ 增加 了固溶体内的结构缺陷, 使得晶格缺陷增加, 导致晶 胞收缩, 晶胞中原子的数目下降, 氧空穴浓度增加, 促进了氧的迁移和扩散, 使得氧和氢的结合能力变 强 ${ }^{[10]}$; 另一方面, $\mathrm{ZrO}_{2}-\mathrm{Al}_{2} \mathrm{O}_{3}$ 载体中存在阳离子缺 陷, 缺陷处的氧离子使缺陷表现出富电子特征, 因此 载体中的缺陷位可被看作聍氢器 ${ }^{[11]}$, 使得其更容易 接受载体表面游离的氢, 以及来自金属 $\mathrm{Pt}$ 溢流的 氢, 从而增强材料的还原性能; (2) $\mathrm{Al}_{2} \mathrm{O}_{3}$ 的加人使得 $\mathrm{SO}_{4}^{2-}$ 在 $\mathrm{ZrO}_{2}$ 上面的分散程度大大地提高了, 从而增 加了 $\mathrm{SO}_{4}^{2-}$ 的还原性 ${ }^{[12]}$; (3) 由于 $\mathrm{Al}_{2} \mathrm{O}_{3}$ 对 $\mathrm{ZrO}_{2}$ 具有吸 电子效应, 减弱了 $\mathrm{SO}_{4}^{2-}$ 与 $\mathrm{ZrO}_{2}$ 之间的相互作用, 从 而削弱了其与 $\mathrm{SO}_{4}^{2-}$ 的成键能力 ${ }^{[13]}$. 因此, 随着 $\mathrm{Al}_{2} \mathrm{O}_{3}$ 的增加, $\mathrm{SO}_{4}^{2-}$ 越容易被 $\mathrm{H}_{2}$ 还原. 这个结果与 Wei 等 ${ }^{[14]}$ 的实验结果相吻合.

\subsection{6 不同 $\mathrm{Al}_{2} \mathrm{O}_{3}$ 含量催化剂的 $\mathrm{NH}_{3}-\mathrm{TPD}$ 分析}

周晓龙等 ${ }^{[15]}$ 认为, $\mathrm{Al}$ 是 $\mathrm{SO}_{4}^{2-} / \mathrm{ZrO}_{2}$ 催化剂烷烃 异构化中非常好的促进组分, 并且 $\mathrm{Al}$ 的掺杂能够增 强 $\mathrm{SO}_{4}^{2-} / \mathrm{ZrO}_{2}$ 的酸性质. 图 7 为 $\mathrm{Al}_{2} \mathrm{O}_{3}$ 质量分数为 $0 \% 、 2.5 \%$ 和 $5.0 \%$ 的 $\mathrm{SO}_{4}^{2-} / \mathrm{ZrO}_{2}-\mathrm{Al}_{2} \mathrm{O}_{3}$ 催化剂的 $\mathrm{NH}_{3}-$ TPD 分析曲线. 由图 7 可以看出, 三种催化剂在 200 ${ }^{\circ} \mathrm{C}$ 左右出现宽的脱附峰是由弱的 L-酸位的吸附引 起的. 在 300-500 ${ }^{\circ} \mathrm{C}$ 之间无明显的尖峰, 说明催化剂 的酸强度分布很分散, 并且弱、中、强酸是同时存在 的, 催化剂表面为多种酸强度中心, 并存的复杂体

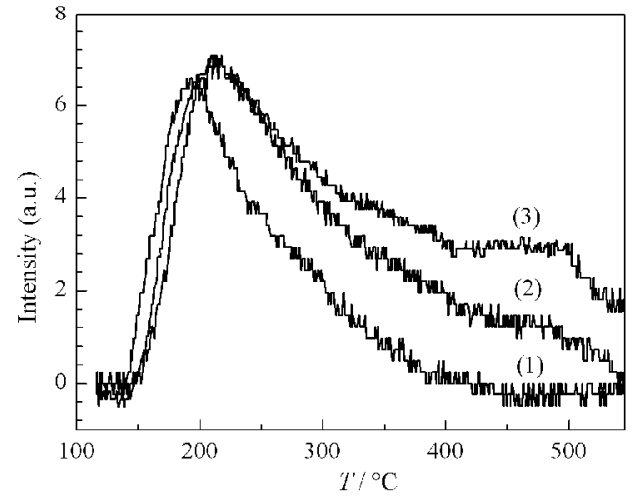

图 7 不同 $\mathrm{Al}_{2} \mathrm{O}_{3}$ 质量分数的 $\mathrm{SO}_{4}^{2-} / \mathrm{ZrO}_{2}-\mathrm{Al}_{2} \mathrm{O}_{3}$ 催化剂的 $\mathrm{NH}_{3}$-TPD 曲线

Fig.7 $\mathrm{NH}_{3}-\mathrm{TPD}$ profiles of $\mathrm{SO}_{4}^{2-} / \mathrm{ZrO}_{2}-\mathrm{Al}_{2} \mathrm{O}_{3}$ catalysts with different $\mathrm{Al}_{2} \mathrm{O}_{3}$ mass fractions $w\left(\mathrm{Al}_{2} \mathrm{O}_{3}\right)$ : (1) $0 \%$; (2) $2.5 \%$; (3) $5.0 \%$

系; 含有铝的催化剂低温脱附峰要比不含铝的催化 剂右移, 说明含铝催化剂的弱酸酸性要比不含铝的 略强; $\mathrm{Al}_{2} \mathrm{O}_{3}$ 质量分数为 $5.0 \%$ 的催化剂在 $500{ }^{\circ} \mathrm{C}$ 左 右有一肩峰, 说明其强酸分布范围很广; $\mathrm{Al}_{2} \mathrm{O}_{3}$ 质量 分数为 $5.0 \%$ 的催化剂在高温区的中强酸的强度要 强于 $\mathrm{Al}_{2} \mathrm{O}_{3}$ 质量分数为 $2.5 \%$ 的催化剂的酸强度, 而 不含铝的催化剂几乎没有中强酸位, 而维持样品反 应活性的是中强酸位 ${ }^{[16]}$. 从氨脱附曲线的峰面积上 可以看出, $\mathrm{Al}_{2} \mathrm{O}_{3}$ 质量分数为 $5.0 \%$ 的催化剂的酸量 均大于 $\mathrm{Al}_{2} \mathrm{O}_{3}$ 质量分数为 $2.5 \%$ 催化剂和不含铝的 催化剂的酸量. 这说明加人一定量的 $\mathrm{Al}$ 不仅提高了 催化剂的酸强度, 而且增加了催化剂的酸总量. 由于 催化剂表面酸性与催化剂表面的硫含量有关, 因此 $\mathrm{Al}_{2} \mathrm{O}_{3}$ 能够提高催化剂酸性的原因可能是由于 $\mathrm{Al}_{2} \mathrm{O}_{3}$ 能够抑制硫酸根的热分解, 这一点与 TG-DTA 分析 表征结果(见图 4)相符合.

\section{2 活性评价}

\subsection{1 不同 $\mathrm{Al}_{2} \mathrm{O}_{3}$ 含量对催化剂的活性评价}

固定浸渍的硫酸物质的量浓度为 $0.5 \mathrm{~mol} \cdot \mathrm{L}^{-1}$, $\mathrm{Pt}$ 质量分数 $0.10 \%, 650{ }^{\circ} \mathrm{C}$ 焙烧, $300{ }^{\circ} \mathrm{C}$ 活化等制备 条件不变, 改变 $\mathrm{Al}_{2} \mathrm{O}_{3}$ 质量分数, 考察其对催化剂性 能的影响, 结果见图 8. 由图 8 可知, $\mathrm{Al}_{2} \mathrm{O}_{3}$ 质量分数 小于 $5.0 \%$ 时, 随 $\mathrm{Al}_{2} \mathrm{O}_{3}$ 质量分数的提高, 异戊烷产 率提高; $\mathrm{Al}_{2} \mathrm{O}_{3}$ 质量分数为 $5.0 \%$ 时, 催化剂对正戊烷 异构化反应的催化活性最好, 在 $220{ }^{\circ} \mathrm{C}$ 时, 异戊烷产 率达到了 $61.3 \%$, 继续提高 $\mathrm{Al}_{2} \mathrm{O}_{3}$ 质量分数, 异戊烷 产率反而下降. 这是因为适量 $\mathrm{Al}_{2} \mathrm{O}_{3}$ 的加人可以提 高表面积(见表 1), 抑制硫的热分解(见图 5), 增强酸 强度(见图7), 有利于提高催化剂的异构化性能; 因 


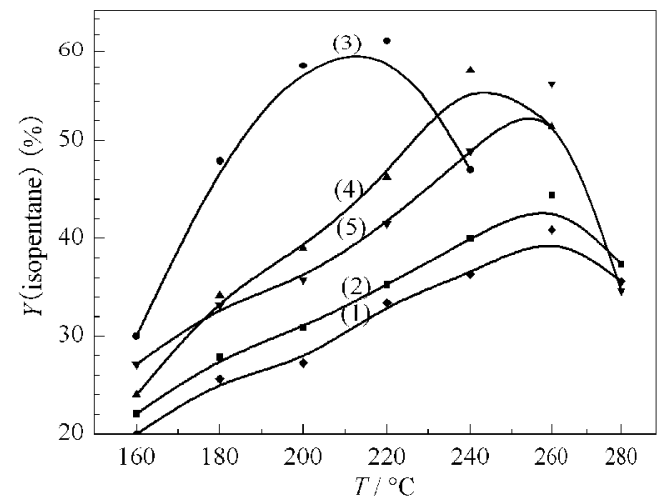

图 8 不同 $\mathrm{Al}_{2} \mathrm{O}_{3}$ 质量分数的 $\mathrm{Pt}-\mathrm{SO}_{4}^{2-} / \mathrm{ZrO}_{2}-\mathrm{Al}_{2} \mathrm{O}_{3}$ 催化剂的异戊烷产率 $(\mathbf{Y})$

Fig.8 Isopentane yield ( $\mathrm{Y}$ ) of $\mathrm{Pt}-\mathrm{SO}_{4}^{2-} / \mathrm{ZrO}_{2}-\mathrm{Al}_{2} \mathrm{O}_{3}$ catalysts with different $\mathbf{A l}_{\mathbf{2}} \mathbf{O}_{3}$ mass fractions $w\left(\mathrm{Al}_{2} \mathrm{O}_{3}\right):$ (1) $0 \%$, (2) $2.5 \%$, (3) $5.0 \%$, (4) $10.0 \%$, (5) $30.0 \%$; $T=300{ }^{\circ} \mathrm{C}, t=3 \mathrm{~h}, p=2.0 \mathrm{MPa}, m\left(\mathrm{H}_{2}\right) / m($ oil $)=4: 1$, WHSV $=1.0 \mathrm{~h}^{-1}$

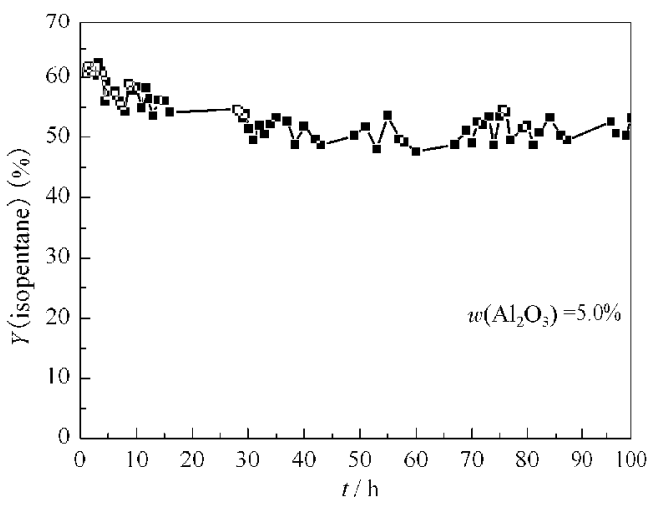

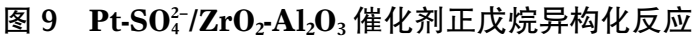
稳定性实验

Fig.9 Stability test of the $\mathrm{Pt}-\mathrm{SO}_{4}^{2-} / \mathrm{ZrO}_{2}-\mathrm{Al}_{2} \mathrm{O}_{3}$ catalyst for pentane isomerization

$T=300{ }^{\circ} \mathrm{C}, t=3 \mathrm{~h}, p=2.0 \mathrm{Mpa}, m\left(\mathrm{H}_{2}\right) / m(\mathrm{oil})=4: 1$, WHSV $=1.0 \mathrm{~h}^{-1}$

$\mathrm{ZrO}_{2}$ 晶体与 $\mathrm{SO}_{4}^{2-}$ 的共同作用而形成超强酸位, 过量 的 $\mathrm{Al}$ 会延迟 $\mathrm{ZrO}_{2}$ 四方晶相的形成(见图 1), 降低 $\mathrm{ZrO}_{2}$ 的质量分数, 催化剂活性中心数目减少, 异构 化活性下降. 所以最佳 $\mathrm{Al}_{2} \mathrm{O}_{3}$ 质量分数为 $5.0 \%$.

\subsection{2 催化剂的稳定性评价}

$\mathrm{Al}_{2} \mathrm{O}_{3}$ 质量分数为 $5.0 \% 、 0.5 \mathrm{~mol} \cdot \mathrm{L}^{-1}$ 硫酸溶液 浸渍、Pt 质量分数 $0.10 \% 、 650{ }^{\circ} \mathrm{C}$ 焙烧, 用 $\mathrm{H}_{2}$ 在 300 ${ }^{\circ} \mathrm{C}$ 下还原 $3 \mathrm{~h}$ 后, 在反应压力为 $2.0 \mathrm{MPa}$ 、氢与烃的摩 尔比 $4: 1$ 、质量空速(WHSV) $1.0 \mathrm{~h}^{-1}$ 不变, 温度 220 ${ }^{\circ} \mathrm{C}$ 下, 连续运行 $100 \mathrm{~h}$, 以考察催化剂的稳定性, 其 结果如图 9 所示. 由图 9 可以看出, 催化剂在 $100 \mathrm{~h}$ 内异戊烷收率稳定, 保持在 52.0\%以上, 此时异戊烷 选择性在 $98.2 \%$ 以上, 表明催化剂具有较好的催化 活性、选择性和稳定性.

\section{3 结 论}

通过向 $\mathrm{SO}_{4}^{2-} / \mathrm{ZrO}_{2}$ 催化剂中同时引人适量的 $\mathrm{Pt}$ 和 $\mathrm{Al}$, 制备出了具有较高催化性能和稳定性的 Pt$\mathrm{SO}_{4}^{2-} / \mathrm{ZrO}_{2}-\mathrm{Al}_{2} \mathrm{O}_{3}$ 型固体超强酸催化剂, 实验表明, $\mathrm{Al}$ 能够延迟 $\mathrm{ZrO}_{2}$ 的晶化温度, 抑制硫的分解; $\mathrm{Al}$ 能够 增加催化剂的比表面积, 增强硫氧键的结合, 提高催 化剂的还原性能, 增加了催化剂的酸强度和酸总量. 当 $\mathrm{Al}$ 质量分数为 $5.0 \%$ 时, $\mathrm{Pt}-\mathrm{SO}_{4}^{2-} / \mathrm{ZrO}_{2}-\mathrm{Al}_{2} \mathrm{O}_{3}$ 固体 超强酸催化剂的催化活性最好, 在反应压力为 2.0 $\mathrm{MPa} 、$ 氢烃摩尔比 $4: 1$ 、质量空速(WHSV) $1.0 \mathrm{~h}^{-1}$ 不变, 温度 $220{ }^{\circ} \mathrm{C}$ 的工艺条件下进行异构化反应, $100 \mathrm{~h}$ 内 异戊烷收率稳定, 保持在 $52.0 \%$ 以上, 此时异戊烷选 择性在 $98.2 \%$ 以上, 表明催化剂具有较好的催化活 性、选择性和稳定性.

\section{References}

1 Jin, T. S.; Yang, M. N.; Feng, G. L.; Li, T. S. Chin. J. Org. Chem., 2003, 23: 1438 [靳通收, 杨米娜, 冯国良, 李同双. 有机化学, 2003, 23: 1438]

2 Reddy, B. M.; Sreekanth, P. M.; Lakshmanan, A. J. Mol. Catal. AChem., 2005, 237: 93.

3 Akira, N.; Hiromi, O.; Yoshikazu, K.; Kiyoshi, O. Catal. Commun., 2005, $6: 716$

4 Gao, Z.; Hua, W. M.; Chen, J. M.; Tang, Y. Acta Phys. -Chim. Sin., 1994, 10: 897 [高滋, 华伟明, 陈建民, 唐 颐. 物理化学学 报, 1994, 10: 897]

5 Pan, H. H.; He, M. Y. Chin. J. Catal., 2005, 26: 1067 [潘轫华, 何鸣元. 催化学报, 2005, 26: 1067]

6 Yao, R. P.; Zhang, M. J.; Yang, J.; Yi, D. L.; Xu, J.; Deng, F.; Yue, Y.; Ye, C. H. Acta Chim. Sin., 2005, 63: 269 [姚瑞平, 张铭金, 杨 俊, 易德莲, 徐 君, 邓 风, 岳 勇, 叶朝辉. 化学学报, 2005, 63: 269]

7 Jin, T.; Yamaguchi, T.; Tanabe, K. J. Phys. Chem., 1986, 90: 4794

8 Vijay, S.; Wolf, E. E.; Miller, J. T.; Kropf, A. J. Appl. Catal. A, 2004, 264: 125

9 Strohmeier, B. R.; Levden, D. E.; Hercules, D. M. J. Catal., 1985, 94: 514

10 Abrahams, I.; Bush, A. J.; Chan, S. C. M. Krok, F.; Wrobel, W. J. Mater. Chem., 2001, 11: 1715

11 Formasiero, P.; Monte, R. D.; Rao, G. J. Catal., 1995, 151: 165

12 Zhou, R. X.; Chen, P.; Zheng, X. M.; Chen, L. S. Acta Phys. -Chim. Sin., 1996, 12: 464 [周仁贤, 陈 平, 郑小明, 陈林深. 物理化 学学报, 1996, 12: 464]

13 Zhou, R. X.; Zheng, X. M. Acta Phys. -Chim. Sin., 1995, 11: 315 [周仁贤, 郑小明. 物理化学学报, 1995, 11: 315]

14 Wei, W. G; Jung, H. W.; Chang, L.; Che, N.; Nan, P. X.; Chung, Y. Catal. Today, 2004, 97: 307

15 Zhou, X. L.; Yu, G. X.; Jin, Y. Q. J. East Chin. Univ. Sci. Technol., 2007, 33: 309 [周晓龙, 余国贤, 金亚清. 华东理工大学学报, 2007, 33: 309 ]

16 Xia, Y. D.; Hua, W. M.; Gao, Z. Acta Chim. Sin., 1999, 57:1325 [夏勇德,华伟明, 高 滋.化学学报, 1999, 57: 1325] 